\title{
Computational Dosimetry
}

B.R.L. Siebert

R.H. Thomas

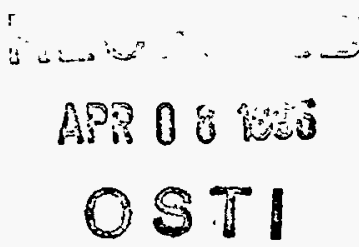

January 1996

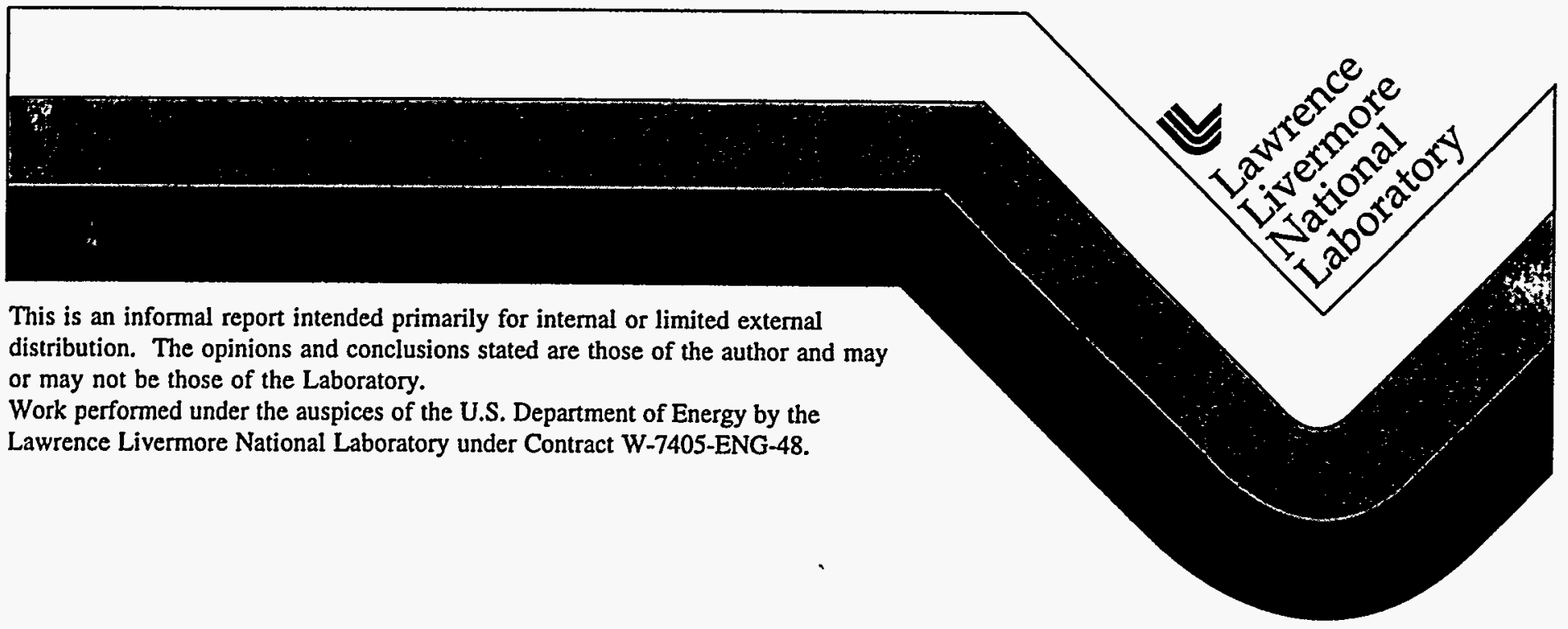




\section{DISCLAIMER}

This document was prepared as an account of work sponsored by an agency of the United States Government. Neither the United States Government nor the University of California nor any of their employees, makes any warranty, express or implied, or assumes any legal liability or responsibility for the accuracy, completeness, or usefulness of any information, apparatus, product, or process disclosed, or represents that its use would not infringe privately owned rights. Reference herein to any specific commercial product, process, or service by trade name, trademark, manufacturer, or otherwise, does not necessarily constitute or imply its endorsement, recommendation, or favoring by the United States Government or the University of California. The views and opinions of authors expressed herein do not necessarily state or reflect those of the United States Government or the University of California, and shall not be used for advertising or product endorsement purposes.

This report has been reproduced directly from the best available copy.

Available to DOE and DOE contractors from the Office of Scientific and Technical Information P.O. Box 62, Oak Ridge, TN 37831

Prices available from (615) 576-8401, FTS 626-8401

Available to the public from the

National Technical Information Service

U.S. Department of Commerce

5285 Port Royal Rd.

Springfield, VA 22161 


\section{Computational Dosimetry}

18 "All the mathematical sciences are founded on relations between physical laws and the laws of numbers, so 19 that the aim of exact science is to reduce the problems of nature to the determination ofquantities by 20 operations with numbers".

ABSTRACT

27 The paper presents a definition of the term "Computational Dosimetry" that is interpreted as the sub28 discipline of computational physics which is devoted to radiation metrology. It is shown that computational

29 dosimetry is more than a mere collection of computational methods. Computational simulations directed at 30 basic understanding and modelling are important tools provided by computational dosimetry, while another 31 very important application is the support that it can give to the design, optimization and analysis of 32 experiments. However, the primary task of computational dosimetry is to reduce the variance in the 33 determination of absorbed dose (and its related quantities), for example in the disciplines of radiological 34 protection and radiation therapy. In this paper emphasis is given to the discussion of potential pitfalls in the 35 applications of computational dosimetry and recommendations are given for their avoidance. The need for 36 comparison of calculated and experimental data whenever possible is strongly stressed. 
INTRODUCTION

The term "Numerical Dosimetry" is puzzling and its meaning requires analysis. That this recently coined term has found its way into general circulation affords yet another valediction of Gresham's Law ${ }^{1}$ The term is of dubious etymological parentage but is nevertheless widely used. Indeed the authors were asked to present a paper on the topic of "Numerical Dosimetry" to this Symposium and wrote an abstract to this title. However, upon reflection the term "Computational Dosimetry" seems a more precise description of the topics covered.

First we must define measurement. Fortunately there is general agreement, supported by dictionaries, that "to measure" is synonymous with "to estimate" and thus, following Maxwell, we infer that dosimetry - the measurement of absorbed dose includes the idea of its calculation. The terminal "-metry" is derived from the Greek $\mu \varepsilon \tau \rho \imath \alpha$ and has the "general sense of action, process or art of measuring (something specified by the initial element)" 2 .

Nevertheless, the attraction of the term "numerical dosimetry" needs explanation before it is dismissed. The authors of this paper speculate that, of the several options offered by the dictionary, those who coined the term would choose either "by numbers" or "expressed by a number or numbers." However, since all determinations of dose, whether by measurement or calculation, are expressed by a number (of units of the quantity) it would seem likely that it is the first of these two options that is intended. We therefore infer that "Numerical Dosimetry" is presumably then the determination of absorbed dose "by numbers". If our speculation is correct this interpretation is indeed .most apt because the detailed mechanisms of most of the radiation transport codes now used for the calculation of dose use random number generators.

The authors prefer to describe these Monte Carlo techniques, and indeed any mathematical solutions to radiation transport problems-which are nowadays basic tools used in the dosimetry of ionising radiation, by the term "Computational Dosimetry".

66 Computational Dosimetry is the sub-discipline of computational physics devoted to radiation metrology. "To compute" derives from the Latin root putare (to clear up or settle) - and from the etymology one can then infer that to compute does not merely mean

\footnotetext{
1 "Bad money drives out good", Sir Thomas Gresham (1558). Some scholars attribute the law to Copernicus.

2 The Oxford English Dictionary adds the explanatory comment- "Most of the subjects in -meter have correlative words in -metry, denoting specifically the process of measuring by the instrument called 'meter'." Thus presumably dosimetry is the determination of dose with a dosemeter.
} 
to put numbers together but rather to place them in order. In that sense, Computational Dosimetry may be defined as the process of connecting and ordering of known data, by means of relations based on theory or established models, in order to create new data and to reveal new insights. The main goal of this paper is to elucidate this process of the acquisition new data by means of computational dosimetry and to question the validity of the knowledge obtained in this way.

It is the contention of the authors that Computational Dosimetry is more than a mere collection of computational methods. This viewpoint is demonstrated by an analysis of the widespread of its use in the papers presented to this Symposium. About half of the papers presented use methods of computational dosimetry. The heavy reliance on Monte Carlo methods is seen from the fact that about 40 papers use transport codes.

This paper addresses the need of basic and derived data as input for computations, shows the role of theory and models in and discusses some subjects of computational dosimetry. The potential pitfalls of computational dosimetry are summarized in the appendix.

BASIC AND DERIVED DATA

The basic data used in dosimetry are atomic and nuclear cross sections for the interactions of interest. In principle data such as kerma coefficients, stopping powers or $\mathrm{W}$-values may be computed from fundamental cross section data and are therefore often referred to as "derived data". In practice, however, the fundamental cross sections are sometimes not known well enough to allow a sufficiently accurate calculation of these derived data and it is often necessary to determine their values by direct measurement .

The need for basic data cross section data is fundamental and much remains to be done in this area(1). Unfortunately the measurement of basic data is tedious, difficult and, in general, expensive. In the present economic climate financial support is dwindling for such measurements and the dearth of information is likely to be with us for some time..

Fortunately several compilations and evaluations of those available cross-section data which are of special interest for neutron dosimetry have been published, together with programs to utilise these evaluated data. Prominent examples are ENDF (2) and NJOY (3), both again available from the Radiation Shielding Information Center, Oak Ridge National Laboratory (RSIC) ${ }^{3}$ or the Nuclear Energy Agency, (NEA) ${ }^{4}$ Both these organizations distribute not only data but also computer codes suitable for dosimetric

\footnotetext{
3Internet: pdc@ornl.gov and WWW: htp://epicws.epm.ornl.gov.

4 Nuclear Energy Agency,

Le Seine St.Germain 12, Boulevard des Iles 92130 Issy-les-Moulinaeux, France;

Internet: sartori@nea.fr
} 
102 applications. So as to ensure the quality assurance of dosimetric determinations it is

103 highly recommended that the assistance of these organizations be sought for information

104 as a means of practising quality assurance of dosimetric determinations. Data for nuclear 105 reactions used in the production of neutron fields are contained in an IAEA Technical 106 Report 273(4). Kerma values are published by the International commission on Radiation 107 Units and Measurements ICRU(5,6), stopping power values by ICRU(7) and Ziegler et $108 a l$. (8) $^{(8)}$ and W-values by the ICRU( $\left.{ }^{9}\right)$. In the field of radiation therapy special attention is 109 drawn to a new IAEA technical document on Atomic and Molecular Data (10). A special 110 source of interaction data are so-called "generators" for high energy particle transport 111 codes. These generators are usually needed as integral parts of such codes, [see, for 112 example, references (11-13), because detailed differential cross section data are still 113 scarce(14).

There are two basic assumptions in the science of radiation dosimetry: first that the fundamental laws of physics apply and second that the absorption of energy by living organisms is the beginning of a complex chain of reactions which are bio-chemicophysical in nature and which may be ultimately either beneficial (e.g., in the case of radiation therapy) or detrimental (e.g., in the case of carcinogenesis).

A theory is a formulation of apparent relationships or underlying principles of certain observed phenomena which has been verified to some degree. A theory therefore serves two purposes: it 'explains' observed data and, by the process of induction, predicts the outcome of potential experiments.

The goal of a complete theory of radiation dosimetry would be to predict the reaction of living organisms and/or the organs of an animal irradiated by ionizing radiation. Such a "dosimetric theory" does not yet exist. Nevertheless computational dosimetry is based on sound theoretical fundamentals. Theoretical input from mathematics, atomic and nuclear physics is used.

Because Monte Carlo techniques are extensively used in Computational Dosimetry one of the most important theoretical considerations is to ensure that the underlying model is physically correct and free from any divergence, even under a variety of complex simulations. The proper application of statistical methods will then permit reliable estimates of the variance of the quantity being calculated, even when a large number of trials are needed to estimate even a small numerical value. 
Models possess some features of a theory but are usually limited in scope. They attempt to describe known data rather than to predict new information: models are not founded on first principles but are merely descriptive. For instance, target theory(15) as applied to the photographic action of X-rays is indeed a theory, however, its numerous offspring applied to biological cells are better termed models.

In the context of this paper the term model is used for the computational image of an experiment, i.e., as general term for all input data and the algorithms used for simulating an experiment. It must be born in mind, that such an image is always an approximation to reality. Apart from technical restrictions such as finite computing resources there are limitations in describing the experimental situation. For instance in depth dose calculations for therapeutic applications there is an uncertainty in anatomical geometries imposed by the finite resolution of the tomographic process. As another example, in calculating response functions of sensors assumptions need to be made on the homogeneity of materials used in its construction which usually cannot be checked by any feasible measurement. As a specific example in this latter regard, small changes in the hydrogen content of materials (caused by adsorption) or slight changes in the content of neutron absorbing substances (e.g., in borated materials), can have strong influence on the neutron moderating properties of the materials comprising the sensor.

In view of these limitations it is clear that all such models are imperfect to some degree. It is necessary to provide a rigorous and balanced assessment of both the merits and limits of any model but, even the most careful discussion can never replace experimental verification. Nevertheless, even bearing these limitations in mind, such models are versatile tools of computational dosimetry. They generate new insights, provide basic understanding and give strong support to the design, optimization and analysis of experiments. In some sense, such models may be regarded as replacing

162 experiments provided they are based on sound assumptions, use correct input data, are 163 carefully checked and are applied within their scope. A simple example which exemplifies these constraints is the calculation of a response function of a radiation detector: if the simulation agrees satisfactorily with measurements in monoenergetic calibration fields, then use of the function at other energies, within the calibration range,

167 is justified. 
171 The primary objective of computational dosimetry is to enhance the accuracy of

172 the determination of absorbed dose. Computational analyses and simulations are the main

173 tools used to achieve these objectives.

174 In this paper we first give examples of the applications of computational 175 dosimetry to radiation-therapy; to the calculation of particle spectra; the calculation of 176 dose distributions; the calculation of instrument response functions and the calculation of 177 conversion coefficients. Following these discussions an example of the optimization of 178 experiments and the tools used to analyse radiation environments are then discussed.

179 Newcomers to computational dosimetry will find the detailed discussion of the 180 MCNP code in ref. (16) (available from RSIC or NEA) and the text on Monte Carlo 181 Techniques by Lux and Koblinger(17), which contains an excellent bibliography. Many 182 useful subroutines for numerical mathematics are to be found in a book be Press et al.,(18) 183 An excellent general text has been written by Paic(19) and, finally, the book by Knoll(20) 184 on radiation sensors is of great value, especially if response functions of detectors are to 185 be calculated.

186 For the casual user, it will usually be most economic and efficient to use standard program packages such as MCNP. However, it is vital that some workers continue to develop new codes so that existing codes are challenged, improvements in accuracy are achieved and "user-friendliness" is to be improved. Furthermore, there is no substitute for the experience and insight gained by writing one's own programs.

Provided adequate computing capacity is available, the reduction of the inherent statistical variance in simulations is nowadays a rather small problem. In any computation a rigorous analysis of variance should always be undertaken. Considerable effort is needed to study the sensitivity of the end-point being calculated with variations of the input parameters. There is no generally agreed method as how best to address the very difficult and complicated problem of the treatment of the variances associated with the input data such as cross sections and values for derived data. For these reasons it is once again stressed that experiments are indispensable to check simulations. exposure to ionizing radiations. The current annual average world-wide effective dose per capita from medical exposures is about $0.6 \mathrm{mSv}$, half of which comes from therapy and the remainder from diagnostic procedures $(21)$. Many diagnostic methods, particularly the generation of images using tomography(22) and therapy-planing(23) rely on highly sophisticated computational methods. Although it is beyond the scope of this paper to give a detailed account of these methods but some general remarks seem appropriate. 
As reported in this conference (e.g., by White et.al.,(23)) modern radiotherapy planing, is a prominent example of the attempt to find a substitute for measurements. Clearly there are many measurements that are impractable in human patients, no matter how important the data required. The alternative, but necessary, task is formidable because an enormous effort in diagnostic-imaging is required to adequately describe the anatomical details, with respect to geometry and material properties, of any specific patient. Even when and if this problem is adequately solved there remains the very basic problem of the verification of such procedures, i.e., the question whether one can design mock ups of the situations encountered in patients which are accessible to measurement and calculation.

The question of the relative biological effectiveness to be applied to the absorbed dose in different organs or tissues is another area for improvement. In order that existing models may be tested, and possibly improved, it will be first necessary to improve the accuracy of calculated particle spectra inside a patient and to complement these with studies on a micrometer, or better nanometer, dosimetric scale which would give insight on track structures and provide a basis for their interaction with the structures of the cells affected by radiation. Here an interdisciplinary approach is required in view of the complex problems encountered in radiation chemistry and biology e.g., references $(24,25)$. Spectra and Dose Distributions. Spectra and dose distributions are needed not only in therapy planning but also in general radiation protection. A knowledge of spectra in the work-place is needed so that select appropriate detectors may be selected to perform radiation monitoring and that the instruments may be properly calibrated.

As a practical example most instruments used for surveying neutron fields are far from ideal. Before attempting a measurement it would be helpful to consult catalogues of typical neutron spectra $(26,27)$ but ultimately the spectrum in the actual site of measurement must be determined.

Spectra for use in calibration and for the determination of the response of neutronmeasuring devices are provided by the International Standards Organization (ISO)(28). It is highly recommended that these data be used so as to provide consistent basis for measurement.

Even small changes in neutron spectra may produce significant changes in instrument readings, therebye increasing the variance of the determination. An example demonstrating this is shown in figure 1 where two estimates of the fission spectrum from bare (unmoderated) ${ }^{252} \mathrm{Cf}$ are shown. In the MCNP code (18) this spectrum is simulated by the two-parameter Watt spectrum. The differences between the two spectra seem very small but, as is shown in Table 1, if these spectra are used to compute the mean neutron 
energy; or fluence-to ambient dose equivalent conversion coefficients, $H^{*}(10) / \Phi$; or the response of Bonner spheres ${ }^{(27)}$ marked differences are encountered.

The use of calculated spectra and the resulting doses to optimize shielding can have significant economic impact. As a rule of thumb, about $20 \%$ of the total construction cost for large accelerators is due to shielding. Excessive shielding which may be necessitated by uncertainties associated with dose estimates can be very costly at these large installations and it is worthwhile to go to considerable effort in refining these calculations. Furthermore, in most countries accurate calculations are required in order to assess the environmental impact of such installations before a permit to construct the new facility. will be granted. Last, not least, spectra provide physical insight which may be helpful or even needed in many applications.

Details on the calculation of absorbed-dose distribution can be found in a recent NCRP report ${ }^{(30)}$ and some additional remarks are given below in context with conversion 255 functions.

256 Response and Conversion Functions. Nowadays, it is good practice to accompany the development of a new detector with a computational simulation of its performance. In this procedure computational images are used to optimize the design of the instrument and then the model is used to compute the response of the detector in several calibration fields in which measurements with the instrument being designed have been made. When good agreement between these calculations and measurements is obtained one can be confident that further calculations of the instrument response may be trusted over the entire range of energy covered by the calibration fields. However, attention must be given to resonances in cross sections of the materials of the detector which may produce anomalous readings in limited energy regions; see, for example ref. (31). To illustrate the use of this technique we select two from the many examples presented in this conference: a new rem counter (31) and a study on the use of a silicon diode as personal dosimeter ${ }^{(32)}$. Both examples clearly demonstrate the benefits of using the methods of computational dosimetry.

So that the numerical values of the protection quantities and operational quantities resulting from irradiation from external sources of ionising radiation may be compared it is necessary to know the distribution of the absorbed dose throughout the actual human body or computational model. When this distribution of the absorbed dose, is known, the appropriate protection and operational quantities may be determined.

276

Considerable effort is required for calculations of the absorbed dose distribution in mathematical computational models. However, modern computing methods (particularly those that use Monte Carlo techniques) have the advantage in that they can 
278 deal with complex irradiation conditions and provide absorbed dose distributions for 279 irradiation in a wide variety of radiation fields. Excellent statistical precision may usually 280 be obtained from such computations but in addition to the statistical uncertainties 281 inherent in such calculations, other uncertainties may arise from several sources (e.g., the 282 data used to simulate radiation interactions with tissue; differences in anatomical 283 modelling; and the inherent variation in human anatomy). In addition, in practical 284 situations, large uncertainties in the estimation of the absorbed dose distributions may 285 arise because off a lack of detailed knowledge of the external radiation field. Although 286 great precision or accuracy is not required in these calculations for the purpose of 287 radiological protection per se it is nevertheless most important to compare the results 288 obtained by several types of calculation - particularly those made in differing 289 computational models and using different computational techniques - so that possible 290 systematic errors may be detected. Such comparisons require appropriate precision in the 291 calculation, even if this precision is not strictly needed for radiological protection.

292 The experimental approach to the determination of these quantities requires construction of a realistic phantom of the human body using tissue-equivalent material 294 and an extensive measurement programme. Accurate measurements require considerable 295 effort and resources and this is particularly true in the case of neutrons. Such resources 296 are generally beyond all but the largest laboratories(33)..Consequently, the number of 297 systematic experimental determinations ${ }^{5}$ of the absorbed dose reported in the scientific 298 literature is limited and resort is usually made to the alternative approach of calculation. 299 The inter-relation between the different quantities used in radiation protection needs a 300 careful analysis ${ }^{(34)}$. Attention to several influences must be given, particularly so in the 301 calculation of conversion coefficients for neutrons $(35)$. The currently recommended 302 conversion factors ${ }^{(36)}$ are expected to be soon superseded by new data based on ICRP 60 303 recommendations. However, new data $H^{*}(10)$ for neutrons are available(37) and in view 304 of the increasing demand for conversion coefficients for $\mathrm{H}^{*}(10)$ for neutrons with high 305 energies we refer to a contribution to this conference(38).

306 Design and Optimization of Experiments. The practical realisation of realistic neutron 307 calibration field is of great practical importance(39). As an example we discuss one 308 example based on a well-designed moderator around a lithium target bombarded by 309 monoenergetic protons. The optimum design requires a compromise between the need to 310 remove heat dissipated in the lithium target and the distortion of the neutron spectrum 311 resulting from moderation by the cooling water. Two designs were discussed. In the one,

5 The term "experimental determination" is used hereto distinguish it from the shorter term " measurement" because throughout this paper the term measurement is taken to include calculation. 
312 the coolant was led through an o-ring forming an annulus of coolant around the

313 circumference of the target and in the other the water was led so that it cooled the entire 314 backing of the target.

315 Table 1 shows that the change in spectra by neutron moderation has a strong influence both on the fluence averaged energies and the ambient dose equivalents(29).

Analysis of Radiation Environments. Finally, we turn to one of the most important tasks of computational dosimetry which is to analyse radiation environments and finding methods to enhance the accuracy of the determination of absorbed dose and dose equivalent.

The program Spektren Bibliothek (SPKTBIB), presented to this conference(25) provides techniques both to improve estimates of dose equivalent $(40)$ and to optimize existing multi-element detectors $(41)$.

SPKTBIB carries out simulations based on catalogued radiation spectra of various environments and predicts the response of dosimeters by calculating sets of conversion coefficients. This technique permits the selection of detectors appropriate for measurement in a given radiation environment with no tedious or expensive experimental effort. However, it must be emphasised that the assumptions used both for the spectra and response functions used in the simulation must be based on experimentally verified data. In that sense SPKTBIB provides a classical example of what computational dosimetry is "all about": Create a computational image relying on experimentally verified pieces of information (spectra, response functions) and substitute experiments by less expensive and faster calculations. The advantage of calculations is, that verified pieces of input can be combined with great ease, to correspond to a multitude of practical situations, whereas an experiment very often provides answers to only one situation at a time.

\section{CONCLUSIONS}

Computational dosimetry is the sub-discipline of computational physics devoted to radiation metrology. It is essential for the analysis and organization of the complex body of data which is needed in dosimetry and it provides models, evaluations and concepts for this purpose.

The ability to use numerical means to simulate practical situations is one of the most powerful tools of computational dosimetry: experimental or clinical set-ups may be represented; particle spectra and dose distributions may be determined; response functions (or matrices) for instruments and conversion coefficients may be calculated. Computational dosimetry greatly facilitates the optimization of experiments and 
supplements the analysis of the data. In appropriate cases Computational Dosimetry can even replace experiments.

These great benefits are not free-thorough training and experience are needed if they are to be obtained. It is necessary to fully understand study the influence of the variance of input data (cross sections and geometry) although this may involve considerable effort when using standard Monte Carlo codes. An important task for the future is to develop standardized procedures to this end.

In summary, Computational Dosimetry greatly enhances the overall quality of radiation dosimetry in therapy and radiation protection. Nevertheless it is important to remember that despite this great utility appropriate experimental verification of any calculated data is nevertheless indispensable!

360 The European Communities have provided generous support to EURADOS and its Working Group IV on Numerical Dosimetry over many years and thus has greatly contributed to the growing use of and experience with the techniques provided by computational dosimetry.

364 One of us (RHT) is grateful to the support of the Hazards Control Department, Lawrence

365 Livermore National Laboratory, University of California, for their gracious hospitality 366 and support.

\section{REFERENCES}

1. Chadwick, M. B. and DeLuca, Jr., P. M. Nuclear Data Needs for Radiation Protection and Therapy Dosimetry. This issue.

2. Rose, P. F. and Dunford, C. L. (editors) ENDF-102 Data Formats and Procedures for the Evaluated Nuclear Data File ENDF-6: BNL-NCS-44945 (National Nuclear Data Center , Brookhaven National Laboratory, Upton,. NY (USA),1990).

3. MacFarlane, R. E., Muir, D.,W. and Boicourt, R. M. The NJOY Nuclear Data Processing System Los Alamos National Laboratory, LA-9303-M, Vol. I, II (1982), Vol. III (1987), Vol. IV (1985). (For the latest version contact RSIC or NEA).

4. IAEA, Handbook on Nuclear Activation Data. Technical ReportsSeries No. 273 (International Atomic Energy Agency, Vienna (Austria), 1987).

5. ICRU, Neutron dosimetry for Biology and Medicine. Report 26 (International Commission on Radiation Units and Measurements, Bethesda, D.C. (USA),1977).

6. ICRU, Photon, Electron, Proton and Neutron Interaction Data for Body Tissues. Report 46 (Bethesda (USA), 1992). 
7. ICRU, Stopping Power and Ranges for Protons and Alpha Particles. Report 49 (Bethesda (USA), 1993).

8 Ziegler, J. F., Biersack, J. P. and Littmark, U. The stopping of Ions in Solids. (Pergamon Press, 1985).

9. ICRU, Average Energy Required to Produce an Ion Pair. Report 31 (Bethesda (USA), 1979).

8. IAEA, Atomic and Molecular Data for Radiotherapy and Radiation Research. IAEATECDOC-799 (Vienna (Austria), 1995).

11. Fassó, A., Ferrari, A.., Ranft, J., Sala, R. P, J., Stevenson, G. R. and Zazula, J.M., FLUKA92.. in Proc. of the Workshop on SimulatingAccelerator Radiation Environments, SARE. Santa Fe, USA, Report LA-12835-C (Palounek, A. (editor),Los Alamos, 1994).

12. Prael, R. E. and Lichtenstein, H. User Guide to LCS: The LAHET Code System, Los Alamos National Laboratoryreport, LA-UR-89-3014 (1989),

13. Mokhov, N., MARS12 Code System. Proc. SARE Workshop, Santa Fe(1993) and MARS 10 Code System: Users Guide. Report FN-509 (Fermi National Accelerator Laboratory, 1989).

14. Konig, A. ., Review of High Energy Data and Model Codes for Accelerator-Based Transmutation, Nuclear Energy Agency,, NEA/NSC/DOC(92)12 (1992).

15. Blau,M. and Altenburger, K. Uber einige Wirkungen von Strahlen, II. Z.Physik, 12, pp. 315-329 (1922)

16 . Briesmeister, J., (Editor): MCNP - A General Monte Carlo Code for Neutron and Photon Transport. Report: LA 7396-M, 1986, (Revised 1991, Los Alamos, USA).

17. Lux, I. and Koblinger, L. MonteCarlo Particle Transport Methods: Neutron and Photon Calculations. Textbook (CRC Press, Boca

Raton, Ann Arbor and Boston, 1991, ISBN 0-8493 6074-9)

18. Press, W.,H., Flannery, B.P., Teukolsky, S.A. and Vetterling, W.T. Numerical Recipes. Textbook (Cambridge University Press, Cambridge, New York, Port Chester, Melbourne and Sidney, 1989, ISBN 052138330 7)

19. Paic, G. (editor), Ionizing Radiation: Protection and Dosimetry. CRC Press, Boca Raton, Florida (USA) 1988, ISBN 0-8493-6713-1)

20. Knoll, G. F. Radiation Detection and Measurement. Textbook (John Wiley \& Sons, New York, Chichester, Brisbane and Toronto, 1979, ISBN 0-471-49545-X)

21. Valentin, J.: UNSCEAR Data Collections on Medical Radiation Exposures: Trends and Consequences. Radiat. Prot. Dosim. 57 pp $85-90$ (1995). 
22. ICRU, Use of Computers in External Beam Radiotherapy Procedures with HighEnergy Photons and Electrons. Report 42 (Bethesda (USA),1987).

23. White, R. M., Chadwick, M. B., Chandler, W. P., Hartmann Siantar, C. L., Resler, D. A., and Weaver, K. A.: High Energy Data and Dose Calculation for Therapy - the PEREGRINE program. This issue.

24. Watt, D. E. and Hill, S. J. A.: An Empirical Model for the Induction of Double Strand Breaks in DNA by the Indirect Action of Radiation. Radiat. Prot-Dosim. 52 , pp. 1720 (1994).

25. Watt, D. E.: Proposals for a Unified System of Bio-Effectiveness and its Consequences in Practice for Radiobiology, Radiation Protection, Particle Therapy and Nuclear Medicine. This issue.

26. Griffith, R.V., Palfalvi, J. and Madhvanath, U.: Compendium of Neutron Spectra and Detectorresponses for Radiation Protection Purposes. Techn. Report Series NO. 318, (IAEA, Vienna 1990).

27. Naismith, O. F. and Siebert, B. R. L.: A database of neutron spectra, instrument response functions, and dosimetric conversion factors for radiation protection applications. This issue.

28. ISO, Neutron Referenence Radiations for Calibrating Neutron- Measuring Devices Used for Radiation Protection Purposes and the Determination of their Response as a Function of Neutron Energy. ISO 8529 (International Standards Organization, Geneva (Switzerland), 1989).

29. Wiegel, B., Alevra, A. V. and Siebert, B. R. L.: Calculations of the Response Functions of Bonner Spheres with a Spherical ${ }^{3} \mathrm{He}$ Proportional Counter using a Realistic Detector Model. (Physikalisch-Technische Bundesanstalt, Braunschweig, PTB Report N-21 (1994), ISBN-3-89429-563-5).

30. NCRP, Conceptual Basis for Calculations of Absorbed-Dose Distributions.. NCRP Report N0. 108 (National Council on Radiation Protection and Measurements, Bethesda (USA), 1991).

31. Burgkhardt, B., Fieg, G., Klett, A., Plewnia, and Siebert, B. R. L.: The Neutron Fluences and $\mathrm{H}^{*}(10)$ Response of the New LB 6411 Remcounter. This issue.

32. Luszik-Bhadra, M., Alberts, W. G., Dietz, E. and Siebert, B. R. L.: Feasibilty Study on an Individual Electronic Neutron Dosemeter.This issue.

33. Tanner, J. E., Piper, R. K., Leonowich, J. A. and Faust, I. G.:Verification of an Effective Dose Equivalent Model for Neutrons. Radiat. Prot-Dosim. 44, pp. 171-174 (1992). 
34. Siebert, B. R. L.: Radiation Quantities: Their Inter-Relationship. Radiat. Prot. Dosim 54 (3-4) (1994) pp. 193-202.

35. Schuhmacher, H. Hollnagel, R. A. and Siebert, B. R. L..: Sensitivity Study of Parameters Influencing Calculations of Fluence-to-Ambient Dose Equivalent Conversion Coefficient for Neutrons. Radiat. Prot. Dosim. 54 , pp. 221-225 (1994)

36. ICRP Publication 51, Data for Use in Protection Against External Radiation. Annals of the ICRP 17 (2,3), Pergamon Press, Oxford, 1987.

37. Siebert, B.R.L. and Schuhmacher, H.: Quality Factors, Ambient and Personal Dose Equivalent for Neutrons, Based on the New ICRU Stopping Power Data for Protons and Alpha particles. Radiat. Prot. Dosim. 58 pp 177-183 (1995).

38. Sannikov, A.V. and Savitskaya, E.N.: Ambient Dose Equivalent Conversion Factors for High Energy Neutrons Based on the New ICRP Recommendations. This issue.

39. Chartier,J. L., Jansky, B., Kluge, H., Schraube, H. and Wiegel, B.:Recent

Developments in the Design, Realization and Specification of realistic Neutron Calibration fields. This issue.

40. Naismith, O.F. and Siebert, B.R.L. and Thomas, D.J.: Response of Neutron

Dosemeters in Radiation Protection Environments and Investigation of Techniques to Improve Estimates of Dose Equivalent. This issue.

41. Alberts, W. G., Dvrschel, B. and Siebert, B. R. L.: Methodical Studies on the Optimisation of Multi-Element Dosemeters in Neutron Fields.This issue.

\section{APPENDIX}

\section{Caveat emptor!}

General Comments on the Use of Computational Dosimetry

- Random number generators are used as backbone of any simulation, but they are never ideal and the statistics of large numbers does not hold for imperfect random generators. So as to minimize the possibility of error the minimum that should be done with any calculation is to use different initial "seeds" and to perform a sequential analysis at least once.

- The Convergence of Monte Carlo calculations must be carefully examined - especially in the case of small calculated end-points. Internal counters should be used in order to obtain some information as to whether they are appropriately sampled. If possible 
analytical checks should be performed. e.g., in computing absorbed doses one should also calculate the first collision dose by analytical means.

- Variance reduction techniques are sometimes unavoidable, e.g., in deep penetration problems. However, one must keep in mind, that these techniques only redistribute the variance. The use of point detectors is recommended for detectors. It may be helpful to

494 check the normalisation by additional track length or surface fluence estimation in a volume around or a surface of at least one point detector.

- In the specification of variances there are two conflicting requirements. On the one hand variances should be given as small as can be justified so that the differences in predictions between alternative procedures may become apparent. On the other hand, if safety is at stake prudence demands that additional safety factors be applied when giving variances.

- The scope and limits of a given model (computational image) with respect to the reliable ranges of arguments and parameters should be discussed. e.g., nuclear resonances, if not properly taken into account, limit the accuracy of interpolations between experimental points using the model.

- Resonances in cross sections need to be carefully considered. In some cases it is important that they be taken into account using the best energy-resolution available e.g.,

510 in computing the transmission through an iron filter at a reactor. It is not usually helpful to specify conversion coefficients at resonance energies.

- Reference data such as provided by IAEA(4), ICRP(36), ICRU (7) or ISO(28) should be used. If other data seem more accurate it is most important to point out the differences

515 between the data used and reference data and to explain the use of other data. The same

516 holds true for the use of evaluated cross sections.

518 - As a minimum a rudimentary sensitivity analysis should be performed by varying some 519 input data, e.g., the radius of a sphere used as estimator for the dose equivalent at a point 520 in the determination of $\mathrm{H}^{*}(10)$ or by selecting cross section data for a given nuclear 521 reaction from different evaluations. 
523 - A variation of input parameters and data is very helpful in gaining more insight and as 524 consistency check. e.g., in studying a moderator assembly the mass-density of one or 525 more cells or regions may be varied (including considering them as voids).

- If at all possible, in important problems should be treated using more then one code. It is most helpful to participate in benchmark problems so that codes may be validated.

- Finally, documentation should present not only the final results. A strong plea is made 531 for sufficient details of the methods and algorithms used, the input data and any 532 intermediate results so that the reader may be able reproduce the published data. e.g., it is 533 helpful to specify the spectral fluence and not merely spectrum weighted data and for 534 instance to document intermediate step in an optimization may help the reader. 

Technical Information Department • Lawrence Livermore National Laboratory University of California - Livermore, California 94551

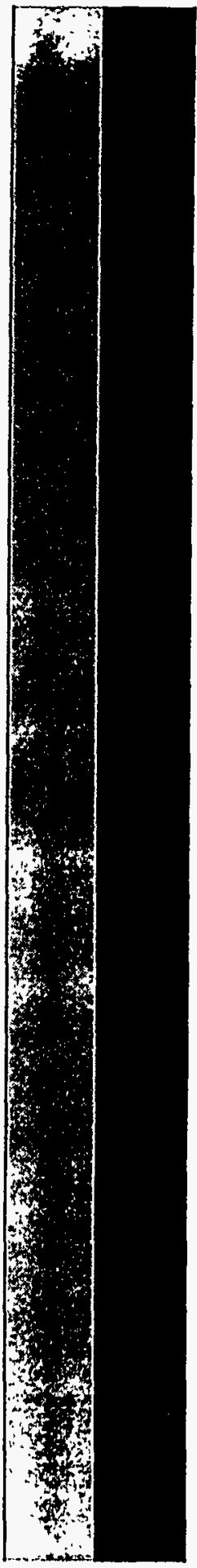

